\title{
Visualization of Hydraulic Cylinder Dynamics by a Structure Preserving Nondimensionalization
}

\author{
Satoru Sakai ${ }^{\circledR}$, Member, IEEE, and Stefano Stramigioli ${ }^{(1)}$, Fellow, IEEE
}

\begin{abstract}
This paper reveals a new simplicity of a nominal hydraulic cylinder model whose original representation suffers from too many physical parameters. The eight-dimensional (8-D) parameter space in the original representation is reduced to a 3-D parameter space in the proposed nondimensional representation while preserving the parametric structure. To clarify comprehensive relations between the nonlinear dynamics and many physical parameters, an advanced direct search approach is suggested. More precisely, we can repeat the fast computation of the nonlinear dynamics and the updates of only three parameters without verifying any new simulator. The efficient visualization of the numerical solutions presents the best possible result corresponding to the analytical solution.
\end{abstract}

Index Terms-Fast computation, hydraulic systems, nonlinear dynamics, visualization.

\section{INTRODUCTION}

B ALANCE between accuracy and simplicity is a key in modeling for control of hydraulic cylinders. Hydraulic cylinders are a fluid-mechanical system. However, instead of the infinite dimensional model, which achieves high accuracy, the finite dimensional nominal models are accepted in many controller design procedures [1]-[4]. Nevertheless, such nominal models are still complex in terms of not only the nonlinear dynamics (nonlinear response) but also many physical parameters in the original representation. In fact, in addition to the well-known mechanical parameters, such as the damping constant, several fluid parameters, such as the bulk modulus and the source pressure, can be dominant in the nonlinear response [5], [6]. Eventually, the comprehensive relations between the nonlinear dynamics and many physical parameters are not entirely clarified. This implies that even if a good control result is achieved under a certain experimental condition, it may not be justifiable to apply the result to more general conditions. For

Manuscript received January 9, 2017; revised May 22, 2017, October 14, 2017, and March 5, 2018; accepted July 5, 2018. Date of publication July 10, 2018; date of current version October 15, 2018. Recommended by Technical Editor J. Mattila. This work was supported in part by the Japan Society for the Promotion of Science KAKENHI JP26420173, and in part by the Fluid Power Technology Promotion Foundation. (Corresponding author: Satoru Sakai.)

S. Sakai is with Faculty of Mechanical Engineering, Shinshu University, Matsumoto 390-8621, Japan (e-mail: satorus@shinshu-u.ac.jp).

$\mathrm{S}$. Stramigioli is with Faculty of Electrical Engineering, University of Twente, Enschede 7522, The Netherlands (e-mail: S.Stramigioli@ utwente.nl).

Color versions of one or more of the figures in this paper are available online at http://ieeexplore.ieee.org.

Digital Object Identifier 10.1109/TMECH.2018.2854751 example, when a linearization-based control [7] is useful for a certain experimental hydraulic system, it is not clear when the linearization is useful again for other hydraulic systems.

To overcome this situation, due to the nonlinear response, numerical studies are important and relevant. However, since the original parameter space is too large due to many physical parameters, it is never efficient to apply the conventional direct search (the brute-force search) approaches [8], in which the computation of the nonlinear dynamics and the updates of many physical parameters are repeated in the original representation. Also, even if the original parameter space is reduced by an usual nondimensional representation, it is never efficient to build and verify a new simulator for the usual nondimensional representation where the existing simulator for the original representation cannot be applied.

On the other hand, such comprehensive relations are already studied for other systems. The mass-damper-spring

$$
m \frac{d^{2} s}{d t^{2}}+d \frac{d s}{d t}+k s=f
$$

in the original representation is transformed to

$$
\ddot{s}^{*}+d^{*} \dot{s}^{*}+s^{*}=f^{*}
$$

in a special nondimensional representation with only one parameter $d^{*}=d / \sqrt{m k}$ preserving the parametric structure and the analytical study completely clarified the relations (e.g., the critical response at $d^{*}=2$ ) based on the linearity. Navier-Stokes equations of a fluid system [9]

$$
\frac{\partial u}{\partial t}+(u \cdot \nabla) u=-\frac{1}{\rho} \nabla p+g e_{g}+\nu \Delta u
$$

in the original representation is transformed to

$$
\frac{\partial u^{*}}{\partial t^{*}}+\left(u^{*} \cdot \nabla^{*}\right) u^{*}=-\nabla^{*} p^{*}+\frac{1}{\operatorname{Fr}^{2}} e_{g}^{*}+\frac{1}{\operatorname{Re}} \Delta^{*} u^{*}
$$

in a special nondimensional representation with only two parameters $\mathrm{Fr}$ and Re preserving the parametric structure and many numerical studies have clarified the relations. These analytical and numerical studies that are not detailed here provide the foundations for many things today.

To clarify such relations for a nominal hydraulic cylinder model as well, this paper proposes a new special nondimensional representation that preserves the parametric structure and suggests an advanced direct search approach different from the conventional ones with respect to both the efficiency and visualization (3-D-visualization), without which many numerical 
studies are less valuable. The proposed nondimensional representation is a new simplicity of the nominal model that does not exist in more accurate or complex existing models (e.g., [10], [11] in the original representation) as well as in the merely simple (freely truncated) existing models. More precisely, without building and verifying any new simulator, we can repeat the fast computation of the nonlinear dynamics and the updates of only three parameters in the proposed nondimensional representation. To provide an example, the numerical existence and nonlinearity are efficiently visualized since the nonlinear dynamics computations are impossible if the numerical existence is not achieved and the linearization is less reliable if the nonlinearity is strong.

The rest of this paper is organized as follows. In Section II, a nominal model of hydraulic cylinders is reviewed in the original representation. A new special nondimensional representation is proposed and compared with other nondimensional representations in Section III. In Section IV, the effectiveness of the proposed nondimensional representation is confirmed. Conclusions are provided in Section V.

\section{NOMINAL MODEL}

Let us start with the nominal model of Fig. 1 in the original representation [12]-[15]

$$
\Sigma_{0}\left\{\begin{array}{l}
M \frac{d^{2} s}{d t^{2}}=-D \frac{d s}{d t}+A_{+} p_{+}-A_{-} p_{-} \\
\frac{d p_{+}}{d t}=\frac{b}{A_{+}(L / 2+s(t))}\left[-A_{+} \frac{d s}{d t}+Q_{+}\left(p_{+}, u\right)\right] \\
\frac{d p_{-}}{d t}=\frac{b}{A_{-}(L / 2-s(t))}\left[+A_{-} \frac{d s}{d t}-Q_{-}\left(p_{-}, u\right)\right]
\end{array}\right.
$$

where the displacement $s(t)[\mathrm{m}]$, the cap pressure $p_{+}(t)[\mathrm{Pa}]$, the rod pressure $p_{-}(t)[\mathrm{Pa}]$, and the spool displacement (the input) $u(t)[\mathrm{m}]$ are the functions of time $t[\mathrm{~s}]$. The subscript + and - denote the cap-side and the rod-side, respectively, and the subscript \pm denotes both sides. The driving force is $f(t)=A_{+} p_{+}(t)-A_{-} p_{-}(t)[\mathrm{N}]$. The mass $M[\mathrm{~kg}]$, the damping constant $D[\mathrm{Ns} / \mathrm{m}]$, the piston areas $A_{+} \geq A_{-}\left[\mathrm{m}^{2}\right]$, and the bulk modulus $b[\mathrm{~Pa}]$ are the positive constants. The cylinder volumes $V_{+}(s(t)):=A_{+}(L / 2+s(t)), V_{-}(s(t)):=$ $A_{-}(L / 2-s(t))\left[\mathrm{m}^{3}\right]$ with the constant stroke $L[\mathrm{~m}]$ are the functions of the displacement $s(t)$. The input flows $Q_{+}$and $Q_{-}$ $\left[\mathrm{m}^{3} / \mathrm{s}\right]$, are approximated by Bernoulli's principle

$$
Q_{+}=B\left(p_{+},+u\right) u, \quad Q_{-}=B\left(p_{-},-u\right) u
$$

with

$$
B(r, u)= \begin{cases}C \sqrt{-r+P} & (u>0) \\ 0 & (u=0) \\ C \sqrt{+r-0} & (u<0)\end{cases}
$$

where the flow gain $C\left[\sqrt{\mathrm{m}^{5} / \mathrm{kg}}\right]$ and the source pressure $P$ $[\mathrm{Pa}]$ are the positive constants. The nominal model introduces the restricted domain $s \in(-L / 2, L / 2)$ and $p_{ \pm} \in[0, P]$ and the absolute notation within the square root functions (2) is dropped.

Remark 1 (Relating to uncertainty): Equation (1) ignores the nonlinear friction effect and also the internal and external leakage effects at least. Equation (2) assumes the steady flow and the negligible servo dynamics of the zero-lapped spool valve.

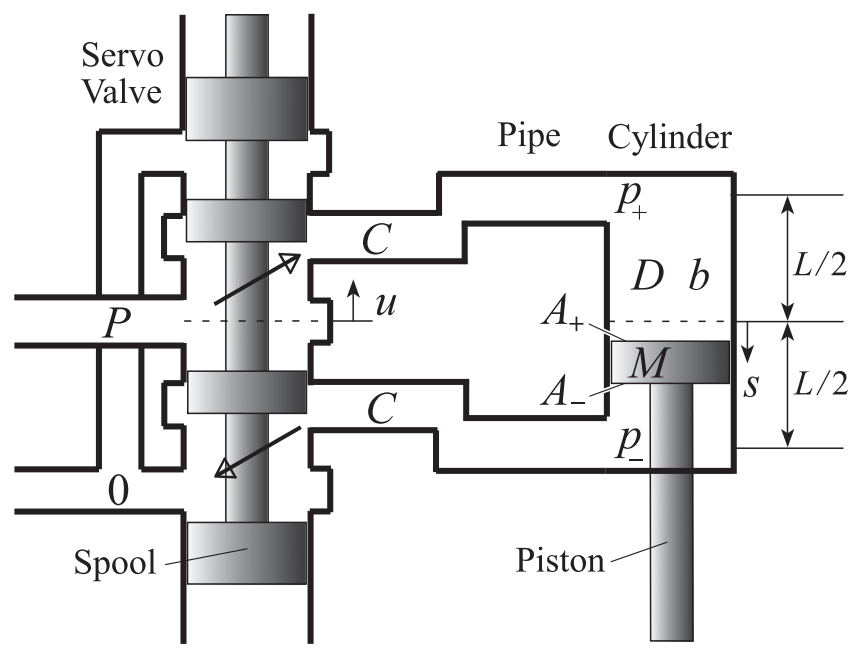

Fig. 1. Nominal hydraulic cylinder model.
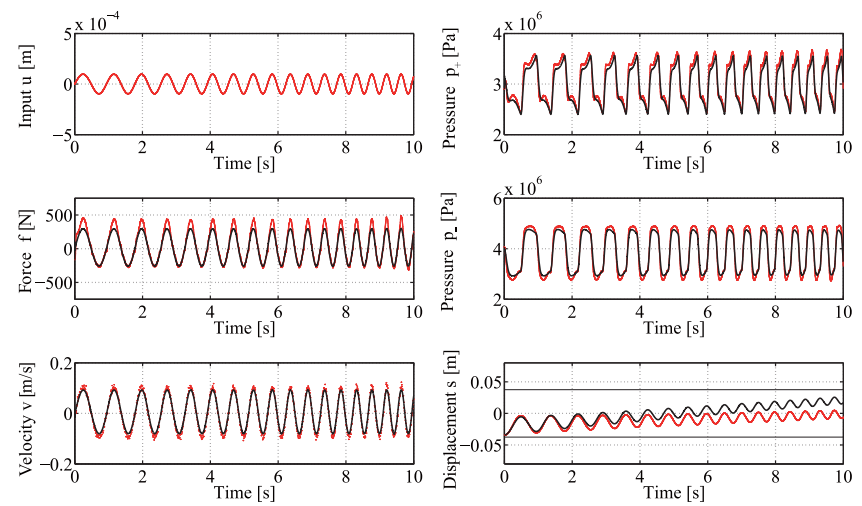

Fig. 2. Example of the nominal model output (the black curves) with $\left(M, D, L, A_{+}, A_{-}, b, C, P\right)=\left(14,3200,0.075,7.0 \times 10^{-4}, 5.4 \times\right.$ $\left.10^{-4}, 5.3 \times 10^{8}, 1.6 \times 10^{-4}, 7 \times 10^{6}\right)$ and the experimental output [15] (the red dots) whose valve is replaced by LSVG-01EH-20-WC-A1-10 (Yuken Kogyo).

On the other hand, the stroke $L$ can include the pipeline length effect and the bulk modulus $b$ includes the pipeline (or tube) flexibility effect. Fig. 2 shows an example of the accuracy between the nominal model (1), (2) and an experimental setup (a real system) in a practical frequency band (see [15] for details). This figure displays a long time cross validation in which experimental outputs (the red dots) were never used in the parameter identification procedure for nominal model outputs (the black curves). Nevertheless, with respect to nonlinear responses in the pressures and displacement, the nominal model has an accuracy that any linearized model (transfer function) cannot have. Of course, the difference (e.g., the nonlinear friction effect) between the nominal model and the experimental setup exists and depends on each experimental setup but would change continuously. In the context of robust control [16], [17], the difference is uncertainty taken into account in the controller design procedure.

The nominal model (1), (2) is not our result. Not the accuracy but a new simplicity is our contribution evaluated in terms of the efficiency and visualization. 


\section{Special NONDIMENSIONALIZATION}

First, a new special nondimensional representation is proposed. Second, the advantages of the proposed nondimensional representation are discussed in comparison with other nondimensional ones because they are not unique [18], [19].

Proposition 1 (Special nondimensional representation): Consider the original representation (1), (2) of the nominal model. Then, there exists a set of time scaling $t^{*}=(1 / T) t$, a variable scaling $\left(s^{*}, p_{+}^{*}, p_{-}^{*}\right)^{\mathrm{T}}=\left((1 / S) s,\left(1 / P_{+}\right) p_{+},\left(1 / P_{-}\right) p_{-}\right)^{\mathrm{T}}$, and an input scaling $u_{*}=(1 / U) u$ by which the original representation (1), (2) is transformed to the following nondimensional representation:

$$
\Sigma_{s}\left\{\begin{array}{l}
\ddot{s}^{*}=-D^{*} \dot{s}^{*}+p_{+}^{*}-A^{*} p_{-}^{*} \\
\dot{p}_{+}^{*}=\frac{1}{1 / 2+s^{*}}\left(-\dot{s}^{*}+Q_{+}^{*}\right) \\
\dot{p}_{-}^{*}=\frac{1}{1 / 2-s^{*}}\left(+\dot{s}^{*}-\frac{1}{A^{*}} Q_{-}^{*}\right)
\end{array}\right.
$$

and

$$
Q_{+}^{*}=B^{*}\left(p_{+}^{*},+u^{*}\right) u^{*}, \quad Q_{-}^{*}=B^{*}\left(p_{-}^{*},-u^{*}\right) u^{*}
$$

with

$$
B^{*}(r, u)= \begin{cases}\sqrt{-r+P^{*}} & (u>0) \\ 0 & (u=0) \\ \sqrt{+r-0} & (u<0)\end{cases}
$$

where $T, S, P_{+}, P_{-}$, and $U$ are the constants and $D^{*}, A^{*}$, and $P^{*}$ are the nondimensional parameters. The notation $\dot{\bullet}$ denotes the derivative with respect to the nondimensional time $t^{*}$.

In the following proof of Proposition 1, the original representation (1), (2) is converted into an input-state equation of a physical form from which the special nondimensional representation (3), (4) is derived via a set of the state and input transformation [20] and also the time transformation.

Proof: The original representation (1), (2) is converted into an input-state equation of the form [13]

$$
\left\{\begin{array}{c}
\frac{d x}{d t}=\underbrace{\left[\begin{array}{cccc}
0 & +1 & 0 & 0 \\
-1 & -D & J_{23} & J_{24} \\
0 & -J_{23} & 0 & 0 \\
0 & -J_{24} & 0 & 0
\end{array}\right]}_{F} \nabla_{x} H+\underbrace{\left[\begin{array}{c}
0 \\
0 \\
+b V_{+}^{-1} Q_{+} \\
-b V_{-}^{-1} Q_{-}
\end{array}\right]}_{g u} \\
y=g^{\mathrm{T}} \nabla_{x} H
\end{array}\right.
$$

with the state $x=\left(s, p_{m}, p_{+}, p_{-}\right)^{\mathrm{T}}$

$$
J_{23}(s)=+b V_{+}(s)^{-1} A_{+}, \quad J_{24}(s)=-b V_{-}(s)^{-1} A_{-}
$$

and the original energy

$$
H=p_{m}^{2} /(2 M)-V_{+}(s)\left(b+p_{+}\right)-V_{-}(s)\left(b+p_{-}\right) .
$$

Here, the notation $\nabla_{x}$ denotes the gradient with respect to the variable $x$. The variable $p_{m}=M v$ is the momentum imparted by the velocity $v=\frac{d s}{d t}$. By the gradient of the original energy $H$ in the input-state equation of the form (5), (1) is obtained by a direct calculation.

Since the state $x$ is defined, let us take the set of time transformation $t^{*}=(1 / T) t$ with $T=\sqrt{(M L) /\left(b A_{+}\right)}=: T_{s}$, the state transformation $x^{*}=\left(s^{*}, v^{*}, p_{+}^{*}, p_{-}^{*}\right)^{\mathrm{T}}=X_{s}^{-1} x$ with

$$
X_{s}:=\left[\begin{array}{cccc}
S & 0 & 0 & 0 \\
0 & M S / T & 0 & 0 \\
0 & 0 & P_{+} & 0 \\
0 & 0 & 0 & P_{-}
\end{array}\right]=\left[\begin{array}{cccc}
L & 0 & 0 & 0 \\
0 & \sqrt{M L b A_{+}} & 0 & 0 \\
0 & 0 & b & 0 \\
0 & 0 & 0 & b
\end{array}\right]
$$

and the input transformation $u_{*}=(1 / U) u$ with $U=$ $\left(\sqrt{A_{+}^{3} L / M}\right) / C=: U_{s}$. Then, the original form (5) is transformed to the nondimensional form

$$
\left\{\begin{array}{c}
\dot{x}^{*}=\underbrace{\left[\begin{array}{cccc}
0 & +1 & 0 & 0 \\
-1 & -D^{*} & J_{23}^{*} & J_{24}^{*} \\
0 & -J_{23}^{*} & 0 & 0 \\
0 & -J_{24}^{*} & 0 & 0
\end{array}\right]}_{T_{s} X_{s}^{-1} F X_{s}^{-T}} \nabla_{x^{*}} H^{*}+\underbrace{\left[\begin{array}{c}
0 \\
0 \\
+J_{23}^{*} Q_{+}^{*} \\
-\left(J_{24}^{*} / A^{*}\right) Q_{-}^{*}
\end{array}\right]}_{T_{s} X_{s}^{-1} g U u^{*}=: g^{*} u^{*}} \\
y^{*}:=g^{* \mathrm{~T}} \nabla_{x^{*}} H^{*}
\end{array}\right.
$$

with

$$
J_{23}^{*}=+1 /\left(1 / 2+s^{*}\right), \quad J_{24}^{*}=-1 /\left(1 / 2-s^{*}\right)
$$

and the nondimensional energy

$$
\begin{aligned}
H^{*}= & (1 / 2)\left(v^{*}\right)^{2} \\
& -\left(1 / 2+s^{*}\right)\left(+1+p_{+}^{*}\right) \\
& -\left(1 / 2-s^{*}\right)\left(+1+p_{-}^{*}\right) A^{*}
\end{aligned}
$$

in which $D^{*}:=D \sqrt{L /\left(M b A_{+}\right)}, A^{*}:=A_{-} / A_{+}, P^{*}:=P / b$. Again, by the gradient of the nondimensional energy $H^{*}$ in the input-state equation of the nondimensional form (6), the nondimensional representation (3), (4) is obtained.

In general, a model is valuable when the model has desirable properties that other models do not have. Not only accuracy but also simplicity for control are among the properties . In a word, this paper highlights that the nominal model has high simplicity that more accurate or complex existing models (e.g., [10], [11], [21]) do not have as well as merely simple (freely truncated) existing models do not. A simplicity for the control of the nominal model is the form (5), which is not our contribution and an application [13] of the physical form [22], [23] developed for the finite-dimensional version of physical systems. The physical form provides so many links to fruitful results in modeling and control (e.g., modeling of infinite-dimensional systems, robust stabilization, learning) [24]-[26] than the general nonlinear forms (e.g., $\dot{x}=f(x)+g(x) u$ and $y=h(x)$ [27]). Indeed, the physical form is a special case of the general nonlinear forms. Regarding our contribution, the rest of this paper reveals that the nominal model has another simplicity for the parametric structure linked to several advantages, that is, the efficiency and visualization.

Technically speaking, the proposed nondimensional representation (3), (4) is different from the conventional ones with respect to the visualization (3-D-visualization) at a minimum. The time scaling in famous nondimensionalizations [21] is not coupled with the state and input scaling to reduce parameters for the visualization. Unlike the translational joint corresponding to the nominal model, the rotational joint [28] is complex due to 
more parameters making the visualization impossible. Also, the translational joint formulation cannot be a special case of the rotational joint formulation [29].

The first advantage of the proposed nondimensional representation (3), (4) is the parameter space reduction. The eightdimensional (8-D) parameter space with $\theta:=\left(M, D, L, A_{+}\right.$, $\left.A_{-}, b, C, P\right) \in \mathbb{R}_{+}^{8}$ in the original representation (1), (2) is reduced to a 3-D parameter space with $\theta^{*}:=\left(D^{*}, A^{*}, P^{*}\right) \in$ $\mathbb{R}_{+} \times(0,1] \times \mathbb{R}_{+} \subset \mathbb{R}_{+}^{3} \subset \mathbb{R}_{+}^{8}$ in the proposed nondimensional representation (3), (4). Of course, other nondimensional representations bring a similar advantage [18], [19]. To discuss the additional advantages of the proposed nondimensional representation (3), (4), let us make our examples of other nondimensional representations.

Example 1: For the original physical form (5), let us take the set of the time transformation $t^{*}=(1 / T) t$ with $T=\sqrt{(M L) /\left(b A_{+}\right)}=: T_{1}$ and the state transformation $x^{*}=$ $\left(s^{*}, v^{*}, p_{+}^{*}, p_{-}^{*}\right)^{\mathrm{T}}=X_{1}^{-1} x$ with

$$
X_{1}:=\left[\begin{array}{cccc}
S & 0 & 0 & 0 \\
0 & M S / T & 0 & 0 \\
0 & 0 & P_{+} & 0 \\
0 & 0 & 0 & P_{-}
\end{array}\right]=\left[\begin{array}{cccc}
L & 0 & 0 & 0 \\
0 & \sqrt{M L b A_{+}} & 0 & 0 \\
0 & 0 & b & 0 \\
0 & 0 & 0 & b A_{+} / A_{-}
\end{array}\right]
$$

and the input transformation $u_{*}=(1 / U) u$ with $U=$ $\left(\sqrt{A_{+}^{3} L / M}\right) / C=: U_{1}$. Then, via a procedure similar to the one in the proof of Proposition 1, we obtain one of the other nondimensional representations

$$
\Sigma_{1}\left\{\begin{array}{l}
\ddot{s}^{*}=-D^{*} \dot{s}^{*}+p_{+}^{*}-p_{-}^{*} \\
\dot{p}_{+}^{*}=\frac{1}{1 / 2+s^{*}}\left(-\dot{s}^{*}+Q_{+}^{*}\right) \\
\dot{p}_{-}^{*}=\frac{1}{1 / 2-s^{*}}\left(+A^{*} \dot{s}^{*}-\frac{1}{\sqrt{A^{*}}} Q_{-}^{*}\right)
\end{array}\right.
$$

and

$$
Q_{+}^{*}=B^{*}\left(p_{+}^{*},+u^{*}\right) u^{*}, \quad Q_{-}^{*}=B^{*}\left(p_{-}^{*},-u^{*}\right) u^{*}
$$

with

$$
B^{*}\left(r, u^{*}\right):= \begin{cases}\sqrt{-r+P^{*}} & \left(u^{*}>0\right) \\ 0 & \left(u^{*}=0\right) \\ \sqrt{+r-0} & \left(u^{*}<0\right)\end{cases}
$$

in which $D^{*}:=D \sqrt{L /\left(M b A_{+}\right)}, A^{*}:=A_{-} / A_{+}, P^{*}:=P / b$.

A significant difference between the nondimensionalizations in Proposition 1 and Example 1 is a parametric structure. By dropping the superscript $\bullet *$, the proposed nondimensional representation (3), (4) can be equal to the original representation (1), (2) when $\theta=(1, D, 1,1, A, 1,1, P) \in \mathbb{R}_{+}^{8}$. However, by dropping the superscript $\bullet *$, one of the other nondimensional representations (7), (8) generally cannot be equal to the original representation (1), (2). More precisely, the first equation in (7) can be equal to the first equation in (1) when $M=A_{+}=A_{-}=1$. The second equation in (7) can be also equal to the second equation in (1) when $L=A_{+}=b=1$. However, even if $L=A_{-}=b=1$, the third equation in (7) cannot be equal to the third equation in (1), since $A^{*} \not \equiv 1$ for any $C$ and $P$. In this sense, the other nondimensional representation
(7), (8) fails to preserve the parametric structure in the original representation (1), (2), whereas the proposed nondimensionalization (3), (4) preserves it successfully. This difference can also be easily observed in the energy. The nondimensional energy in the other nondimensional representation $(7),(8)$ is described by

$$
\begin{aligned}
H^{*}= & (1 / 2)\left(v^{*}\right)^{2} \\
& -\left(1 / 2+s^{*}\right)\left(+1+p_{+}^{*}\right) \\
& -\left(1 / 2-s^{*}\right)\left(+1+p_{-}^{*} / A^{*}\right) A^{*}
\end{aligned}
$$

and the parametric structure differs from that of the original energy $H$ since $A^{*} \not \equiv 1$.

Eventually, the above significant difference is trivially rephrased as the following time response property of the hydraulic cylinders. Let the notation $\phi[\theta, x(0), u(t)]$ denote the state $x(t)$ in the original representation (1), (2) of $\theta=(M, D, L$, $\left.A_{+}, A_{-}, b, C, P\right)$ at time $t$ starting from the initial state $x(0)$ in the presence of the input signal $u(\tau)(0 \leq \tau \leq t)$.

Theorem 1 (Structure preserving property): Suppose the state $x(t)=\phi[\theta, x(0), u(t)]$ exists. Then, the nondimensional state $x^{*}\left(t^{*}\right)=X_{s}^{-1} \phi\left[\theta, X_{s} x^{*}(0), U_{s} u^{*}\left(T_{s} t^{*}\right)\right]$ in the special nondimensional representation (3), (4) at nondimensional time $t^{*}=\left(1 / T_{s}\right) t$ starting from the nondimensional initial state $x^{*}(0)=X_{s}^{-1} x(0)$ in the presence of the nondimensional input $u^{*}\left(t^{*}\right)=\left(1 / U_{s}\right) u\left(t^{*}\right)$ is given as

$$
x^{*}\left(t^{*}\right)=\phi\left[\theta_{\text {special }}^{*}(\theta), x^{*}(0), u^{*}\left(t^{*}\right)\right]
$$

$$
\text { of } \theta_{\text {special }}^{*}(\theta)=(1, \underbrace{D \sqrt{L /\left(M b A_{+}\right)}}_{0<D^{*}}, 1,1, \underbrace{A_{-} / A_{+}}_{0<A^{*} \leq 1}, 1,1, \underbrace{P / b}_{0<P^{*}}) \text {. }
$$

The second advantage is the verification-free based on Theorem 1. The existing simulator for the original representation (1), (2) cannot be applied as a simulator for the other nondimensionalization (7), (8). It is never efficient to build a new simulator for the other nondimensional representation. Moreover, from a practical viewpoint, the verification (e.g., checking of the simulator codes or settings) is more laborious than the building. But, based on Theorem 1, the existing simulator for the original representation (1), (2) is successfully applicable as a simulator for the proposed nondimensional representation (3), (4). Then, we do not have to endure the verification. The third advantage is the fast computation based on Theorem 1. The computation time for $x(t)=X_{s} \phi\left[\theta_{\text {special }}^{*}(\theta), x^{*}(0), u^{*}\left(t / T_{s}\right)\right]$ should be shorter than that for $x(t)=\phi[\theta, x(0), u(t)]$. This is because the number of multiplication and division operations for the forward dynamics computations of (3), (4) is trivially small due to unity parameters $\left(M, A_{+}, L, b, C\right)=(1,1,1,1,1)$ in (9), whereas the parametric structure in the original representation (1), (2) is preserved. Of course, computer performances have much improved since 1990' [29]. However, depending on the objective (e.g., numerical study or design optimization), the computation time is still substantial when many dynamics computations need to be repeated.

The fourth advantage of the proposed nondimensional representation is discussed after our next example.

Example 2. For the original physical form (5), let us take the set of time transformation $t^{*}=(1 / T) t$ with $T=\sqrt{M /(P L)}$ 
$=: T_{2}$ and the state transformation $x^{*}=\left(s^{*}, v^{*}, p_{+}^{*}, p_{-}^{*}\right)^{\mathrm{T}}=$ $X_{2}^{-1} x$ with

$$
X_{2}:=\left[\begin{array}{cccc}
S & 0 & 0 & 0 \\
0 & M S / T & 0 & 0 \\
0 & 0 & P_{+} & 0 \\
0 & 0 & 0 & P_{-}
\end{array}\right]=\left[\begin{array}{cccc}
L & 0 & 0 & 0 \\
0 & \sqrt{M P L^{3}} & 0 & 0 \\
0 & 0 & P & 0 \\
0 & 0 & 0 & P
\end{array}\right]
$$

and the input transformation $u_{*}=(1 / U) u$ with $U=$ $\left(\sqrt{L^{7} / M}\right) / C=: U_{2}$. Then, via a procedure similar to the one in the proof of Proposition 1, we obtain one of the other nondimensional representations

$$
\Sigma_{2}\left\{\begin{array}{l}
\ddot{s}^{*}=-D^{*} \dot{s}^{*}+A_{+}^{*} p_{+}^{*}-A_{-}^{*} p_{-}^{*} \\
\dot{p}_{+}^{*}=\frac{b^{*}}{1 / 2+s^{*}}\left(-\dot{s}^{*}+\frac{1}{A_{+}^{*}} Q_{+}^{*}\right) \\
\dot{p}_{-}^{*}=\frac{b^{*}}{1 / 2-s^{*}}\left(+\dot{s}^{*}-\frac{1}{A_{-}^{*}} Q_{-}^{*}\right)
\end{array}\right.
$$

and

$$
Q_{+}^{*}=B^{*}\left(p_{+}^{*},+u^{*}\right) u^{*}, \quad Q_{-}^{*}=B^{*}\left(p_{-}^{*},-u^{*}\right) u^{*}
$$

with

$$
B^{*}\left(r, u^{*}\right):= \begin{cases}\sqrt{-r+1} & \left(u^{*}>0\right) \\ 0 & \left(u^{*}=0\right) \\ \sqrt{+r-0} & \left(u^{*}<0\right)\end{cases}
$$

in which $D^{*}:=D / \sqrt{M P L}, A_{+}^{*}:=A_{+} / L^{2}, A_{-}^{*}:=A_{-} / L^{2}$, $b^{*}:=b / P$

Now, the other nondimensionalization (10), (11) also preserves the parameter structure in the original representation (1), (2). Indeed, by dropping the superscript $\bullet *$, the other nondimensional representation (10), (11) can be equal to an original representation (1), (2) when $\theta=\left(1, D, 1, A_{+}, A_{-}, b, 1,1\right)$. The nondimensional energy of the other nondimensional representation (10), (11) described by

$$
\begin{aligned}
H^{*}= & (1 / 2)\left(v^{*}\right)^{2} \\
& -\left(1 / 2+s^{*}\right)\left(b^{*}+p_{+}^{*}\right) A_{+}^{*} \\
& -\left(1 / 2-s^{*}\right)\left(b^{*}+p_{-}^{*}\right) A_{-}^{*}
\end{aligned}
$$

can be a special case of the original energy $H$.

The fourth advantage of the proposed nondimensional representation is the visualization. A difference between the nondimensionalizations in Proposition 1 and Example 2 is the dimension of the parameter space. Of course, the 4-D parameter space with $\left(D^{*}, A_{+}^{*}, A_{-}^{*}, b^{*}\right) \in \mathbb{R}_{+}^{4}$ is much smaller than the original 8-D parameter space $\mathbb{R}_{+}^{8}$ and close to the proposed 3-D parameter space with $\theta^{*}=\left(D^{*}, A^{*}, P^{*}\right)$. However, only the proposed 3-D parameter space can be visualized in 3-D, whereas even the 4-D parameter space cannot.

\section{3-D VISUALIZATION}

Not the accuracy but the new simplicity is evaluated in terms of the efficiency (parameter space reduction, verification-free, and fast computation) and visualization.

\section{A. Numerical Existence and Nonlinearity}

For many practical nonlinear systems, one of the most fundamental properties may be the stability [30] as long as the state exists. Especially, it is relevant that the state $x(t)$ exists within an restricted region: $\Omega_{L P}=(-L / 2, L / 2) \times \mathbb{R} \times[0, P]^{2}$ in the presence of the input. In addition to the numerical existence, the nonlinearity (or the input-output linearity) is also of interest for the linearization-based controls [7], [31].

The numerical existence is evaluated by the existence of the escape time $t_{e}$ [30] at which the state $x\left(t_{e}\right)$ starting from a test initial state $x(0)=\left(0,0, P / 2,\left(A_{+} / A_{-}\right) P / 2\right)^{\mathrm{T}} \in \Omega_{L P}$ leaves the region $\Omega_{L P}$ for the first time in the presence of a test signal $u(t)=A_{u} \sin \left(2 \pi f_{u} t\right)$ in the test period $\left[0, T_{u}\right]$. The numerical existence is achieved only if the escape time $t_{e} \in\left[0, T_{u}\right]$ does not exist. The numerical existence depends on the setting of the test parameters $A_{u}, f_{u}$, and $T_{u}$ as well as $\theta=\left(M, D, L, A_{+}, A_{-}, b, C, P\right)$ and $x(0)$.

The nonlinearity is evaluated by a difference between an output of the nominal model and that of the linearized model

$$
\hat{\Sigma}_{0}\left\{\begin{array}{l}
M \frac{d^{2} \hat{s}}{d t^{2}}=-D \frac{d \hat{s}}{d t}+A_{+} \hat{p}_{+}-A_{-} \hat{p}_{-} \\
\frac{d \hat{p}_{+}}{d t}=b V_{+}(0)^{-1}\left[-A_{+} \frac{d \hat{s}}{d t}+Q_{+}(P / 2, u)\right] \\
\frac{d \hat{p}_{-}}{d t}=b V_{-}(0)^{-1}\left[+A_{-} \frac{d \hat{s}}{d t}-Q_{-}(P / 2, u)\right]
\end{array}\right.
$$

whose state $\hat{x}(t):=\left(\hat{s}(t), \dot{\hat{s}}(t), \hat{p}_{+}(t), \hat{p}_{-}(t)\right)^{\mathrm{T}}$ starts from the same state $\hat{x}(0)=x(0)$ in the presence of the same input $u(t)$. The displacement $s(t)$ and the driving force $f(t)=A_{+} p_{+}(t)-$ $A_{-} p_{-}(t)$ are relevant in control [1], [7], whereas the pressures $p_{ \pm}(t)$ are used in parameter identification [15]. Here, the difference is defined as the FIT ratio [32]

$$
\operatorname{FIT}\left(y_{0}^{i}\right)=\left(1-\frac{\sqrt{\sum_{t=0}^{T_{e}}\left(\hat{y}_{0}^{i}(t)-y_{0}^{i}(t)\right)^{2}}}{\sqrt{\sum_{t=0}^{T_{e}}\left(y_{0}^{i}(t)-\bar{y}_{0}^{i}\right)^{2}}}\right) \times 100
$$

where $\bar{y}_{0}^{i}$ is the mean value of the $i$ th element $y_{0}^{i}(t)(i=1, \ldots, 4)$ of the outputs $y_{0}(t):=\left(p_{+}(t), p_{-}(t), f(t), s(t)\right)^{\mathrm{T}}$ of the nominal model and $\hat{y}_{0}^{i}$ is the $i$ th element of the corresponding outputs $\hat{y}_{0}(t):=\left(\hat{p}_{+}(t), \hat{p}_{-}(t), \hat{f}(t), \hat{s}(t)\right)^{\mathrm{T}}$ of the linearized model (12). The results on the velocity $v(t)$ can be discussed by that on the displacement $s(t)$. If the numerical existence is achieved, $T_{e}:=T_{u}$, otherwise $T_{e}:=t_{e} \in\left[0, T_{u}\right]$. The value of $\operatorname{FIT}\left(y_{0}^{i}\right)$ can be negative.

\section{B. Experimental Conditions}

The nonlinear dynamics computation and the parameter updates were repeated in the proposed nondimensional representation instead of the original representation. For nonlinear dynamics computations, (9) was applied to compute the nondimensional state $x^{*}\left(t^{*}\right)$ starting from the initial state $x^{*}(0)=\left(0,0, P^{*} / 2, A^{*} P^{*} / 2\right)^{\mathrm{T}}$ in the presence of the test signal $A_{u}^{*} \sin \left(2 \pi f_{u}^{*} t^{*}\right)$ with the amplitude $A_{u}^{*}:=A_{u} / U_{s}=0.01$ and the frequency $f_{u}^{*}:=T_{s} f_{u} \in[0.001,10]$. The test period was defined as $\left[0, T_{u}^{*}\right]:=\left[0,5 / f_{u}^{*}\right]$. The modified backward differential formula with the variable step was applied (20-sim, 
Ver. 4.1, 64-b $2.60 \mathrm{GHz}$ CPU with $8 \mathrm{~GB}$ of memory). The nondimensional outputs $y_{0}^{*}\left(t^{*}\right)$ were given by the nondimensional state $x^{*}\left(t^{*}\right)$ directly. Using a similar procedure, correspondingestimated outputs $\hat{y}_{0}^{*}\left(t^{*}\right)$ were also given by linearized model (12) in the nondimensional version. The damping constant, the rod area, and the source pressure $\left(D^{*}, A^{*}, P^{*}\right) \in\left[D_{\min }^{*}\right.$, $\left.D_{\max }^{*}\right] \times\left[A_{\min }^{*}, A_{\max }^{*}\right] \times\left[P_{\min }^{*}, P_{\max }^{*}\right]=[0.0006,11.2] \times[0.5$, $1.0] \times\left[1.4 \times 10^{-5}, 0.07\right]$ were updated with the increments $\delta_{D}^{*}=1.12, \delta_{A^{*}}=0.05$, and $\delta_{P^{*}}=0.007$, respectively, and the other parameters $\left(M, A_{+}, L, b, C\right)=(1,1,1,1,1)$ were not updated.

\section{Experimental Results and Discussion}

Fig. 3 shows the numerical existence and nonlinearity visualized in $D^{*} A^{*} P^{*}$ space. In the colorless regions on the slices, the numerical existence is not achieved since the escape time $t_{e}^{*} \in\left[0, T_{u}^{*}\right]$ exists, such that $x^{*}\left(t_{e}^{*}\right) \notin \Omega_{1 P^{*}}$. Fig. 4 shows the time response examples corresponding to Fig. 3 in the following four cases.

CASE-a: $\left(D^{*}, A^{*}, P^{*}\right)=(0.0006,0.5,0.07)$;

CASE-b: $\left(D^{*}, A^{*}, P^{*}\right)=(0.0006,0.9,0.07)$;

CASE-c: $\left(D^{*}, A^{*}, P^{*}\right)=(6.0,0.5,0.07)$;

CASE-d: $\left(D^{*}, A^{*}, P^{*}\right)=(6.0,0.9,0.07)$.

The outputs $y_{0}^{*}\left(t^{*}\right)$ are depicted as the curves. The maximum variable step was $10^{4}$ times larger than the minimum one.

The numerical existence was not achieved when $A^{*} \leq 0.5$ at every frequency $f_{u}^{*}$. When $0.5<A^{*} \leq 1.0$, the numerical existence depended on the frequency $f_{u}^{*}$. In particular, at the low frequency $f_{u}^{*} \leq 0.001$, the numerical existence was not achieved for $0.07 \leq P^{*}$. This may not be surprising in the sense that $P^{*}$ increases only the gain of the nondimensional transfer function matrix

$$
\frac{\sqrt{8 P^{*}}}{p^{2}+D^{*} p+2\left(1+A^{*}\right)}\left[\begin{array}{c}
\left(+p^{2}+D^{*} p-2\left(1-A^{*}\right)\right) /(2 p) \\
\left(-p^{2}-D^{*} p-2\left(1-A^{*}\right)\right) /\left(2 A^{*} p\right) \\
p+D^{*} \\
1 / p
\end{array}\right]
$$

from the input $u^{*}$ to the estimated outputs $\hat{y}_{0}^{*}=\left(\hat{p}_{+}^{*}, \hat{p}_{-}^{*}, \hat{f}^{*}\right.$, $\left.\hat{s}^{*}\right)^{\mathrm{T}}$ of the linearized model (12) in the nondimensional version. The increase of $P^{*}$ corresponds to the increase of the amplitude of the test signal. Here, the notation $p$ denotes the derivative operator in the Laplace transform with respect to the nondimensional time $t^{*}\left(=t / T_{s}\right)$. Indeed, in Fig. 4(a), there always exists $t_{e}^{*} \leq 450$, such that $x^{*}\left(t_{e}^{*}\right) \notin \Omega_{1 P^{*}}$ due to the displacement saturation $s^{*}\left(t_{e}^{*}\right) \rightarrow+0.5$. Additionally, around the resonance frequency $\hat{f}_{r}^{*}\left(D^{*}, A^{*}\right):=\sqrt{2\left(1+A^{*}\right)-\left(D^{*} / 2\right)^{2}} /$ $(2 \pi) \in(0,1 / \pi)$ of the linearized model when the underdamping $\left(D^{*}<2 \sqrt{2\left(1+A^{*}\right)} \in(2 \sqrt{2}, 4]\right.$, the numerical existence was not always achieved. In Fig. 4(f), there exists $t_{e}^{*} \leq 6.5$, such that $x^{*}\left(t_{e}^{*}\right) \notin \Omega_{1 P^{*}}$ due to the pressure saturation $p_{-}^{*}\left(t_{e}^{*}\right) \rightarrow P^{*}$ in CASE-a.

The colored regions on the slices in Fig. 3 depict the FIT ratio as the nonlinearity. In Fig. 5, which corresponds to Fig. 4, the estimated outputs $\hat{y}_{0}^{*}\left(t^{*}\right)$ are depicted as the curves. For the pressures $p_{ \pm}^{*}(t)$, remarkably, around a frequency $f_{u}^{*}=0.02$, the lower nonlinearity (higher linearity) was achieved uniformly in
$D^{*} A^{*} P^{*}$ space. These were observed as time-response examples in Figs 4(d) and 5(d). At other frequencies, the pressures $p_{ \pm}^{*}\left(t^{*}\right)$ and the estimated ones $\hat{p}_{ \pm}^{*}\left(t^{*}\right)$ could be very different as shown in Figs. 4(b) and 5(b), in spite of the best initial condition $p_{ \pm}^{*}(0)=\hat{p}_{ \pm}^{*}(0)$. In Fig. 2 , these nonlinearities, the non-negative and multipeak pressures in CASE-c of Fig. 4(b), were already observed.

For the driving force $f^{*}\left(t^{*}\right)=p_{+}^{*}\left(t^{*}\right)-A_{-}^{*} p_{-}^{*}\left(t^{*}\right)$, the lower nonlinearity was achieved at every frequency $f_{u}^{*}$ in $D^{*} A^{*} P^{*}$ space uniformly. Figs. 4(c) and 5(c) show the corresponding time response examples. Interestingly, even when the pressures $p_{ \pm}^{*}\left(t^{*}\right)$ and the estimated ones $\hat{p}_{ \pm}^{*}\left(t^{*}\right)$ were very different, the force $f^{*}\left(t^{*}\right)$ could be approximated roughly by the estimated one $\hat{f}^{*}\left(t^{*}\right)$. At every high frequency $10<f_{u}^{*}$, not only the pressure changes $p_{ \pm}^{*}\left(t^{*}\right)$ but also the force $f^{*}\left(t^{*}\right)$ was small, as shown in Figs. 4(h) and 5(h) and the nonlinearity was less important.

For the displacement $s^{*}\left(t^{*}\right)$, the nonlinearity was not uniform in $D^{*} A^{*} P^{*}$ space and also sensitive to the frequency $f_{u}^{*}$. The lower nonlinearity was achieved at every frequency $f_{u}^{*}$ when $0.9<A^{*} \leq 1.0$. As shown in Fig. 4(b) and (c), as long as $A^{*} \neq 1.0$, the displacement $s^{*}\left(t^{*}\right)$ could be asymmetric and was not always approximated by the estimated one $\hat{s}^{*}\left(t^{*}\right)$, which was more symmetric. At a glance, one may think that such asymmetric displacements were generated by a nonlinear friction effect. This conjecture is not true because the nominal model ignores the nonlinear friction effect. At every high frequency $0.1 \leq f_{u}^{*}$ except around the resonance frequency $\hat{f}_{r}^{*}\left(D^{*}, A^{*}\right)$, the displacement $s^{*}\left(t^{*}\right)$ was small, as shown in Figs. 4(h) and $5(\mathrm{~h})$ and the nonlinearity was again less important.

In all, at every frequency $f_{u}^{*}$, the linearization was roughly reliable for the driving force $f^{*}\left(t^{*}\right)$ in all cases and also for the displacement $s^{*}\left(t^{*}\right)$ when $0.9 \leq A^{*} \leq 1.0$. For the pressures $p_{ \pm}^{*}\left(t^{*}\right)$, the linearization was roughly reliable around the frequency $f_{u}^{*}=0.02$ in all cases. Precisely speaking, even for the driving force $f^{*}\left(t^{*}\right)$, the asymmetric nonlinearity existed as long as $A^{*} \neq 1.0$ and will affect the force and position control performance via the linearization. The verification-free and the visualization were successfully evaluated.

Remark 2 (Relating to parameter perturbation): Every parameter perturbation in the original parameter space (e.g., $\left.b \rightarrow b\left(1+\delta_{b}\right)\right)$ can also be the perturbation in $D^{*} A^{*} P^{*}$ space (e.g., $D^{*} \rightarrow D \sqrt{L /\left(M b\left(1+\delta_{b}\right) A_{+}\right)}=: D^{*}\left(1+\delta_{D^{*}}\right), P^{*} \rightarrow$ $\left.P /\left(b\left(1+\delta_{b}\right)\right)=: P^{*}\left(1+\delta_{P^{*}}\right)\right)$ by which a point in $D^{*} A^{*} P^{*}$ space is mapped into the other point. Note that the nonlinearity (color) at these points in Fig. 3 evaluates uncertainty for the linearized model (12) and is different from uncertainty for the nominal model discussed in Remark 1.

For the numerical study to clarify comprehensive relations between the nonlinear dynamics and many physical parameters, the proposed nondimensional representation has only $O\left(n^{3}\right)$ time complexity, whereas the original representation has $O\left(n^{8}\right)$ time complexity. Since our experimental condition took $n=10$ to make Fig. 3, the number of updates of physical parameters was remarkably reduced. For the design optimization [8], which is not our main objective in this paper, since we may need the 5-D search after the 3-D search, the 

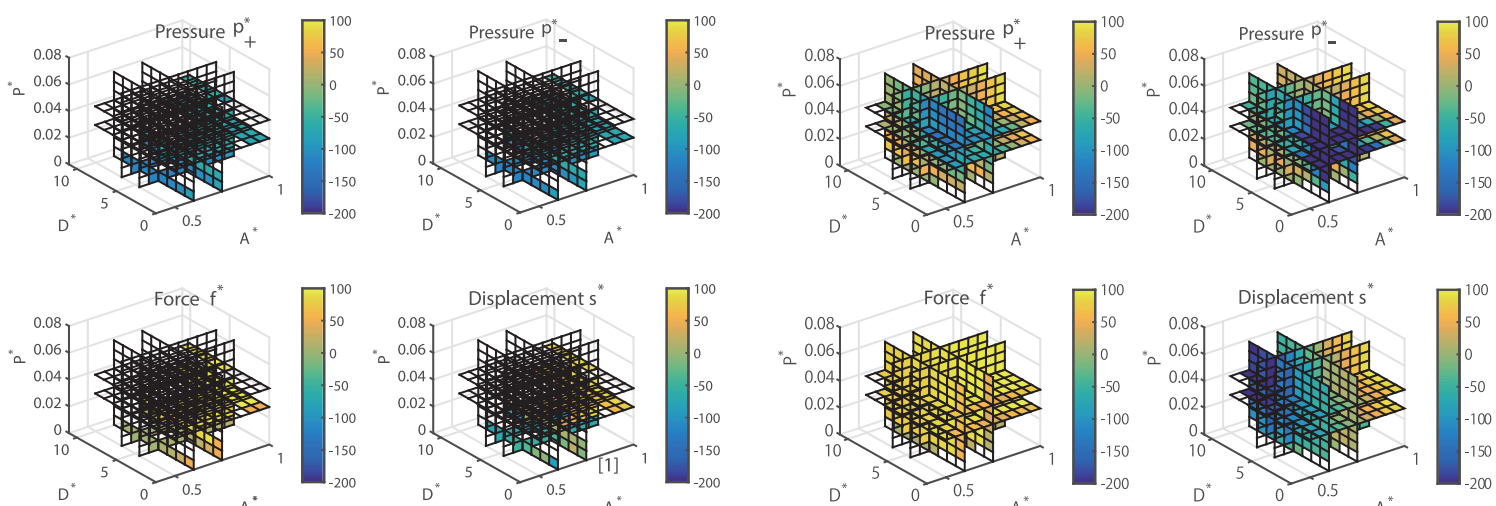

(a)
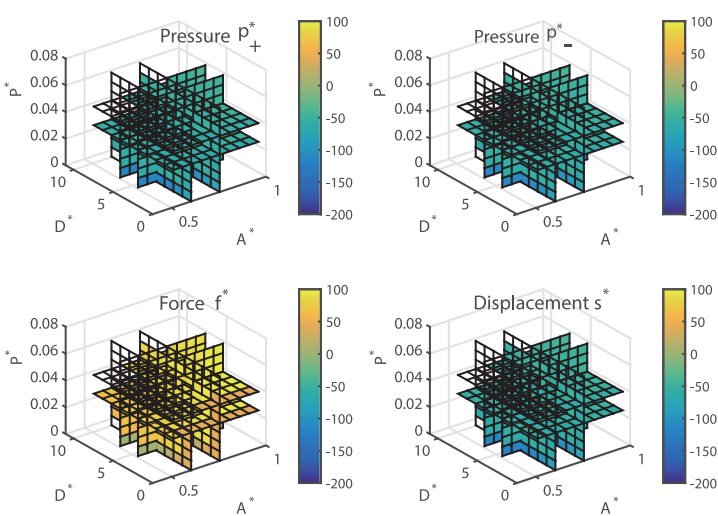

(b)

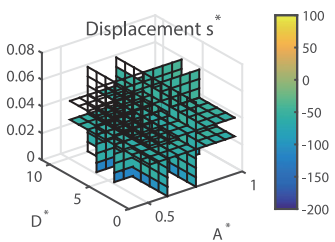

\section{0}
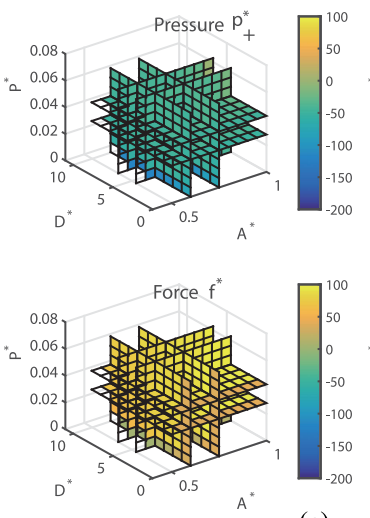

(c)
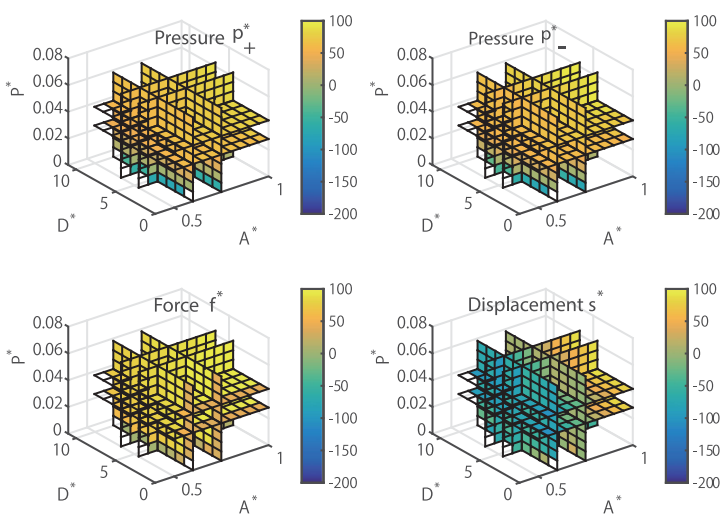

(d)
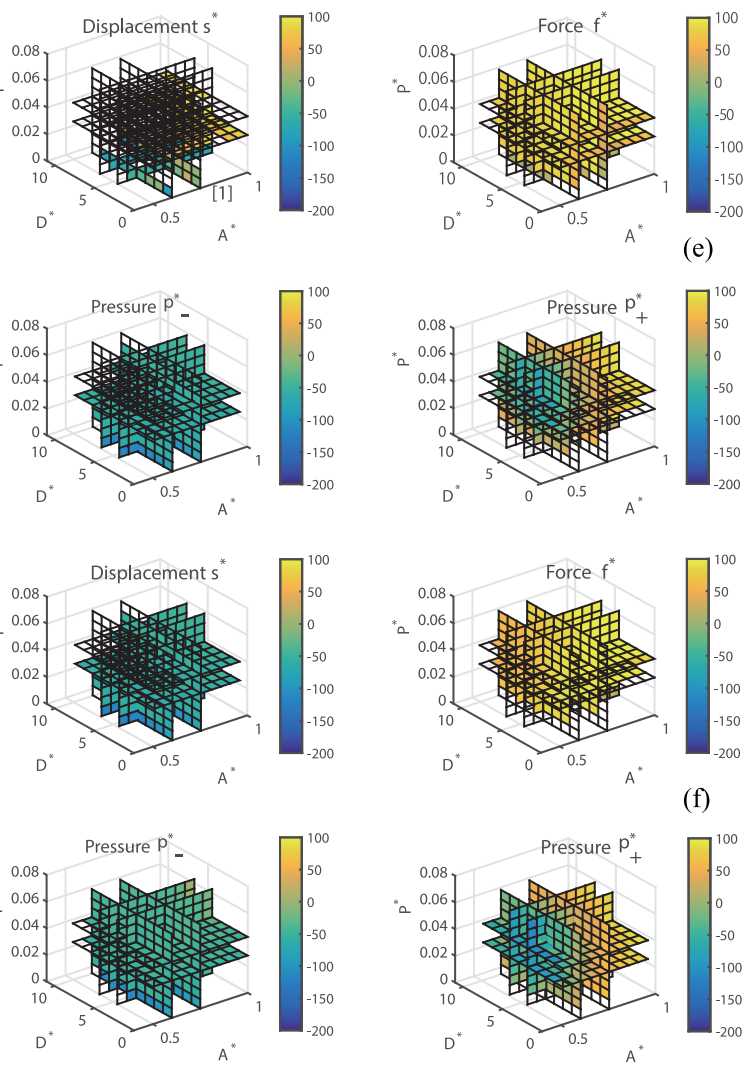

(e)
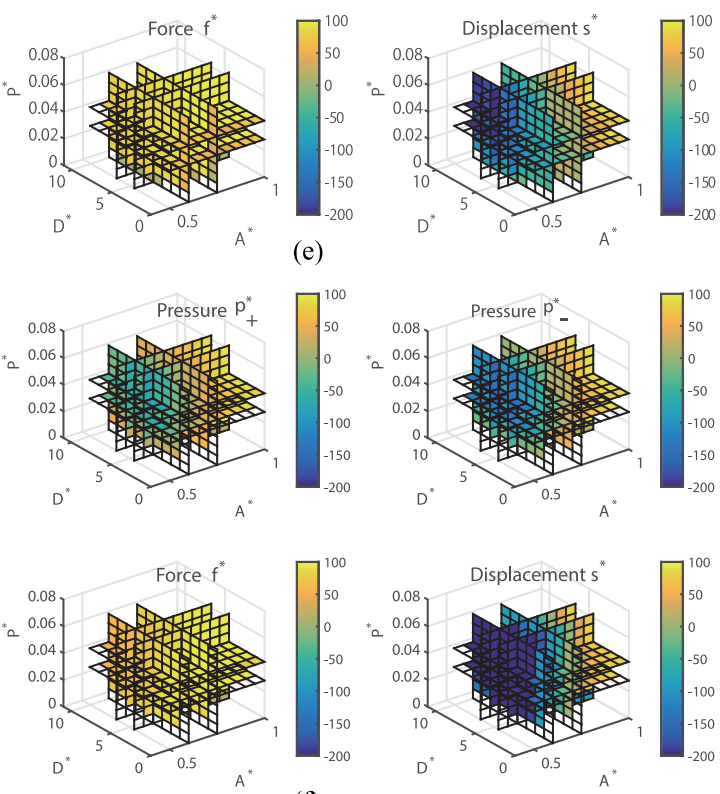

(f)
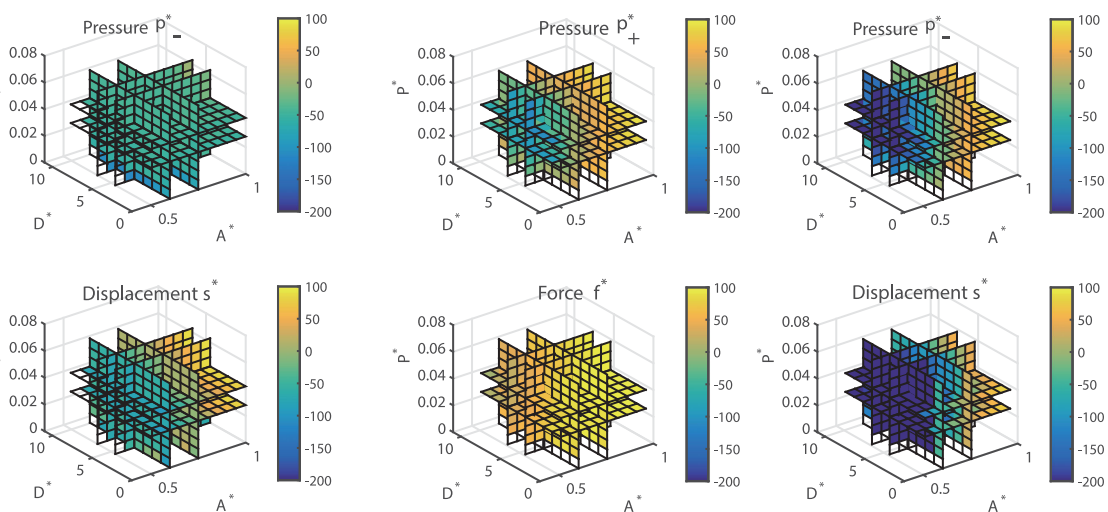

(g)
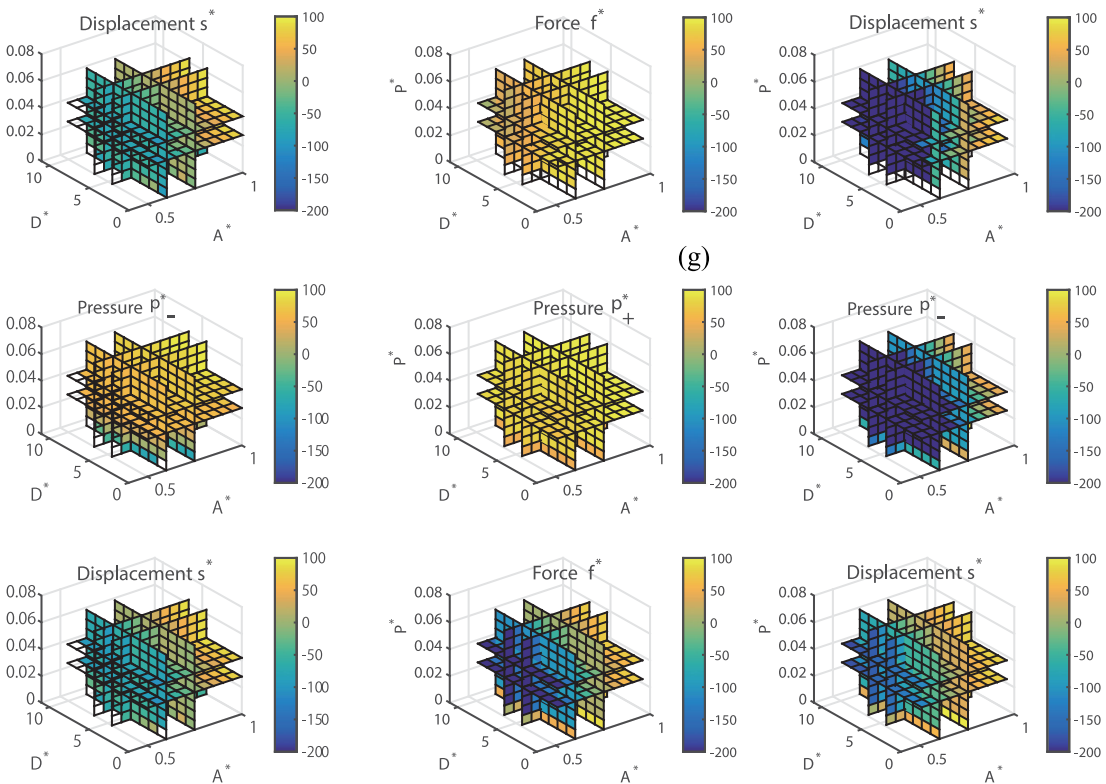

(h)

Fig. 3. Numerical existence and nonlinearity in $D^{*} A^{*} P^{*}$ space. (a) $f_{u}^{*}=0.001$. (b) $f_{u}^{*}=0.002$. (c) $f_{u}=0.003$. (d) $f_{u}^{*}=0.02$. (e) $f_{u}^{*}=0.1$. (f) $f_{u}^{*}=$ 0.3. (g) $f_{u}^{*}=0.5$. (h) $f_{u}^{*}=10$. 

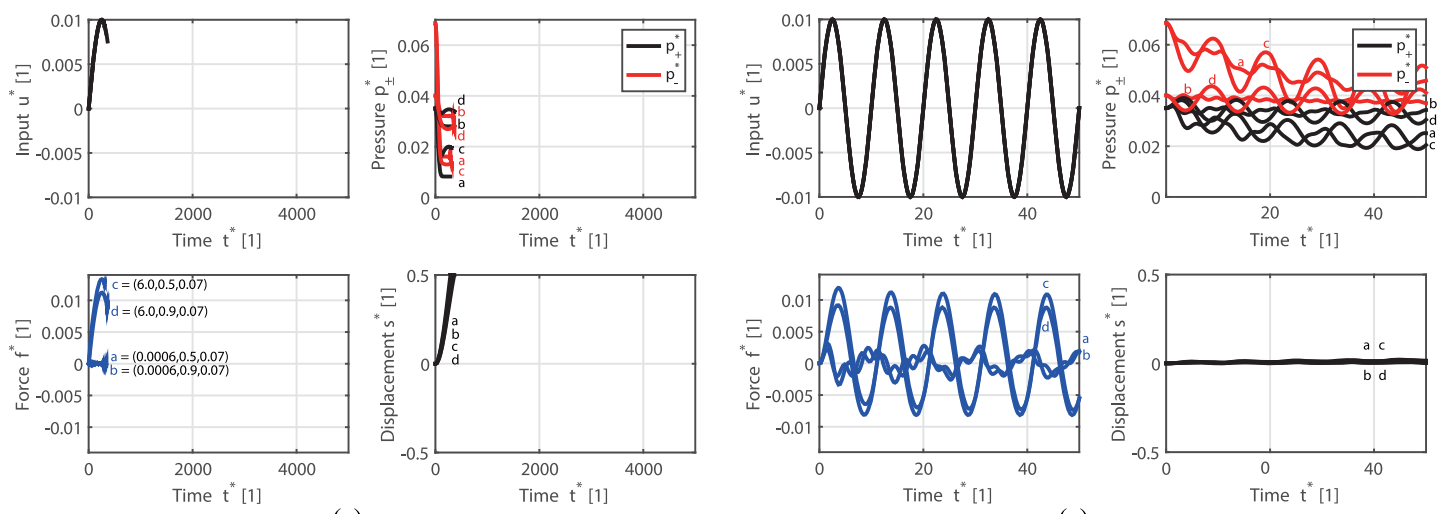

(a)

(e)
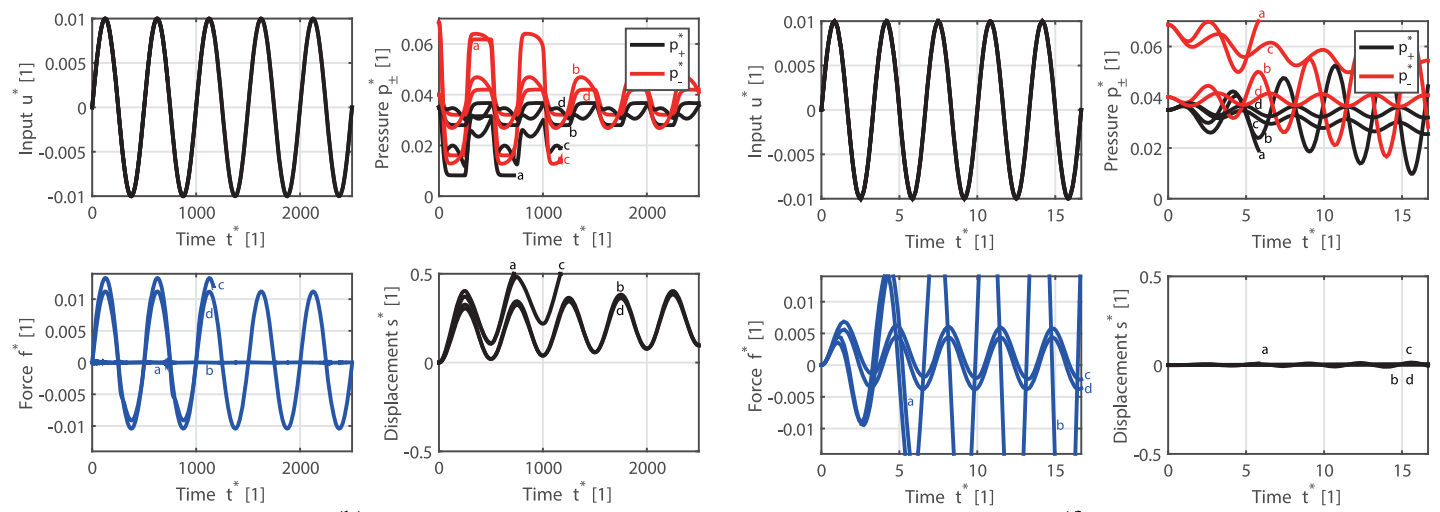

(b)
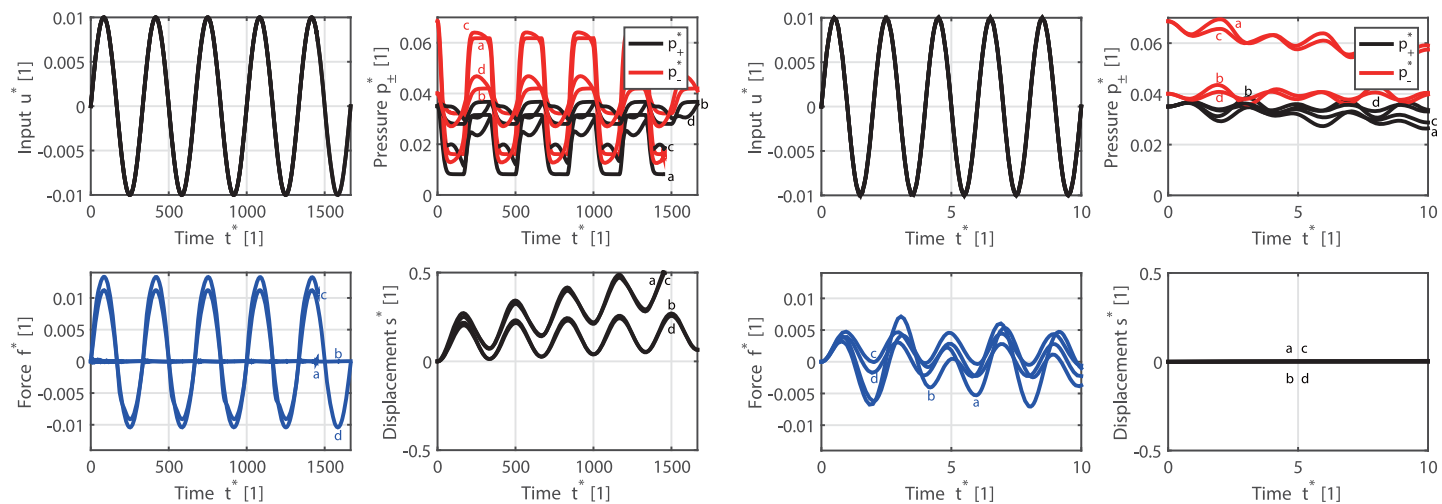

(c)
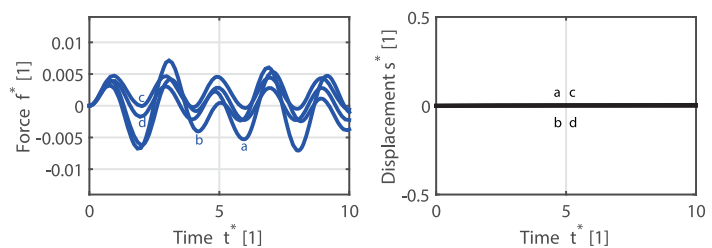

(g)
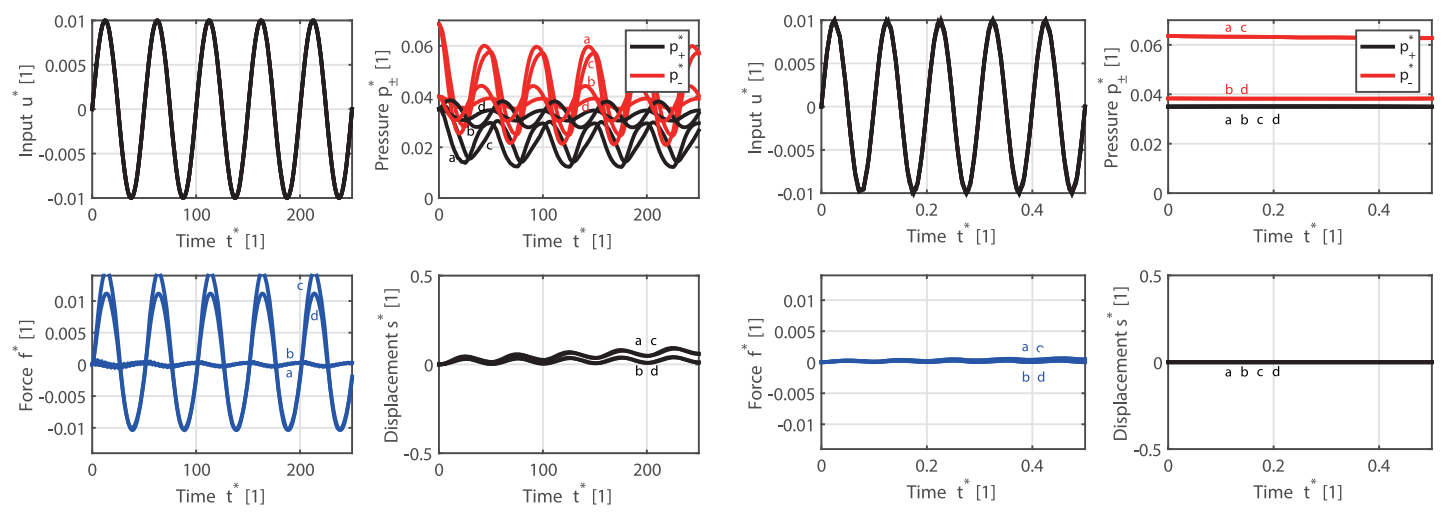

(d)
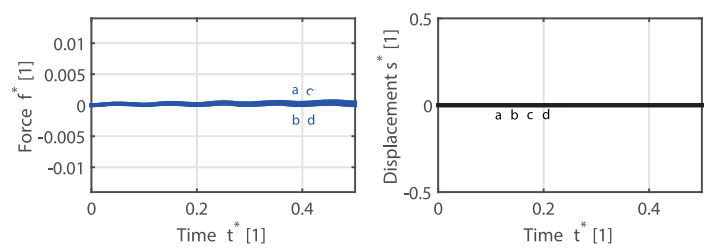

(h)

Fig. 4. Nondimensional time response (Nominal model). (a) $\mathrm{f}_{\mathrm{u}}^{*}=0.001$. (b) $\mathrm{f}_{\mathrm{u}}^{*}=0.002$. (c) $\mathrm{f}_{\mathrm{u}}^{*}=0.003$. (d) $\mathrm{f}_{\mathrm{u}}^{*}=0.02$. (e) $\mathrm{f}_{\mathrm{u}}^{*}=0.1$. (f) $\mathrm{f}_{\mathrm{u}}^{*}=0.3$. (g) $f_{u}^{*}=0.5$. (h) $f_{u}^{*}=10$. 

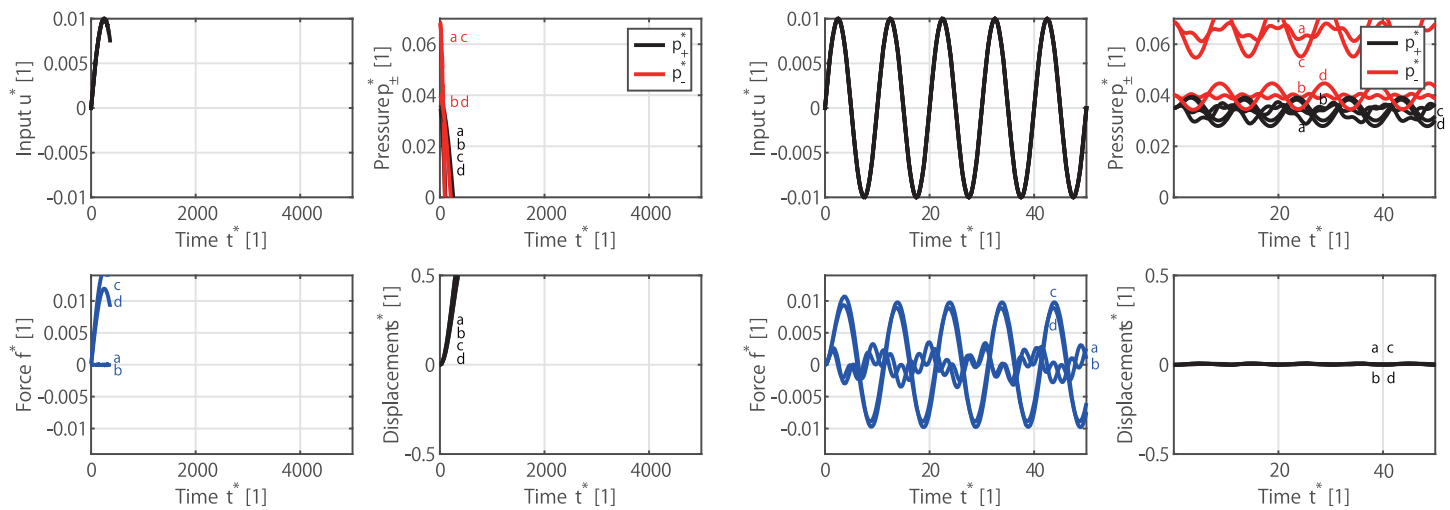

(a)

(e)
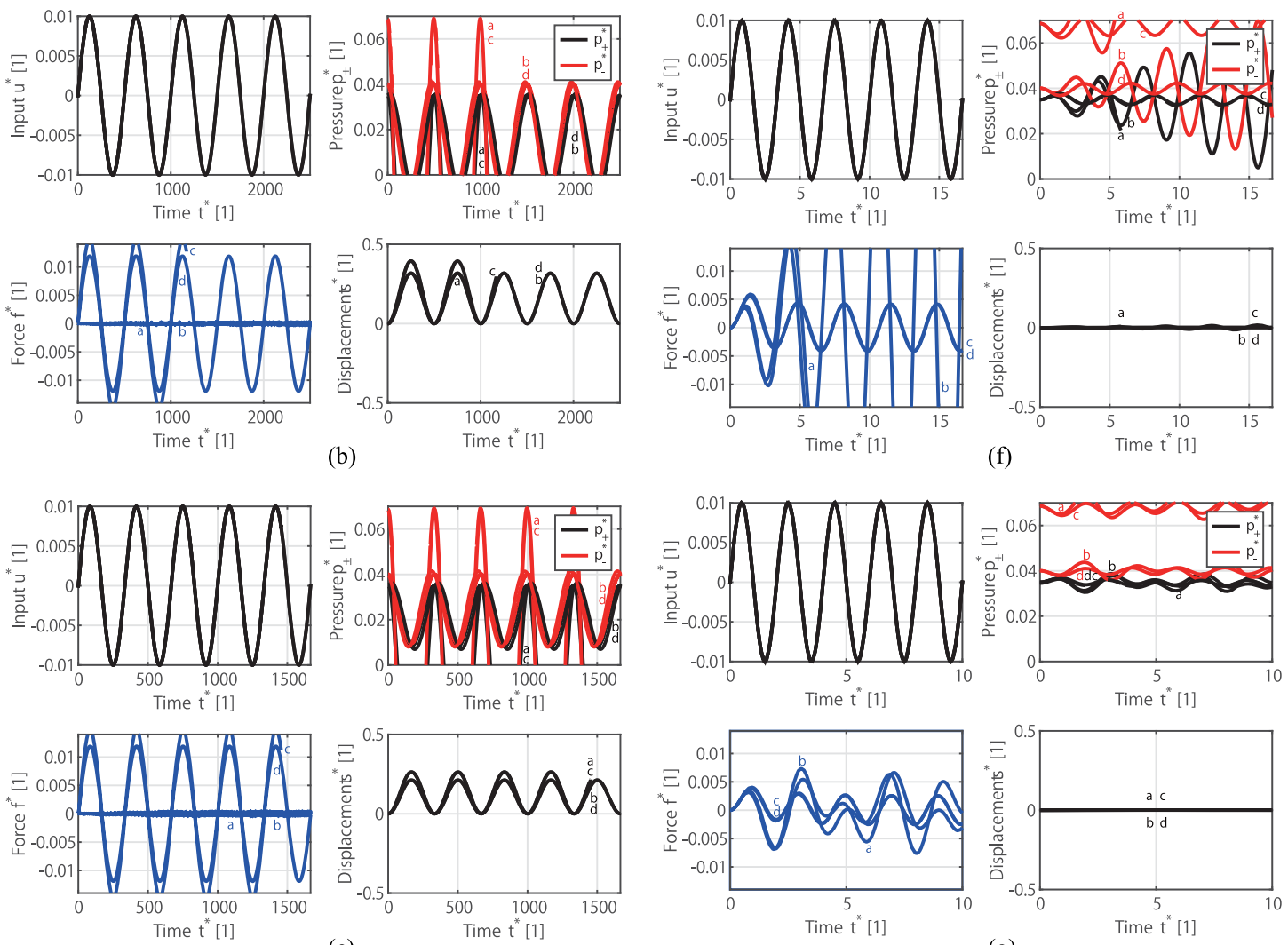

(c)

(g)
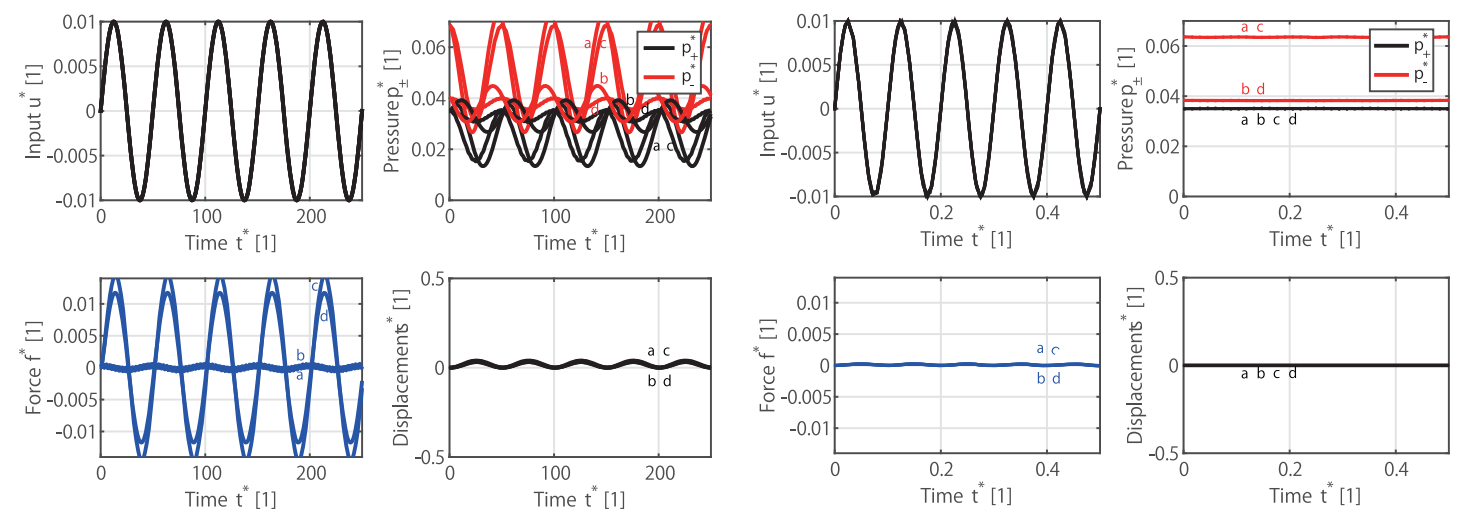

(d)
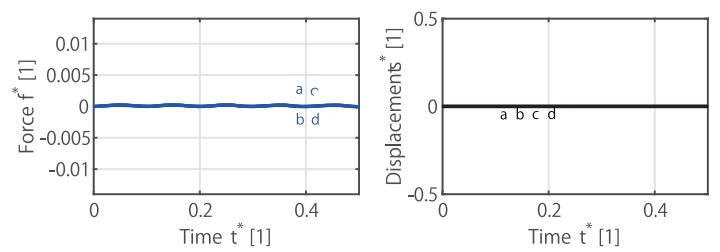

(h)

Fig. 5. Nondimensional time response (Linearized model). (a) $f_{u}^{*}=0.001$. (b) $f_{u}^{*}=0.002$. (c) $f_{u}^{*}=0.003$. (d) $f_{u}^{*}=0.02$. (e) $f_{u}^{*}=0.1$. (f) $f_{u}^{*}=0.3$. (g) $f_{u}^{*}=0.5$. (h) $f_{u}^{*}=10$. 
TABLE I

COMPUTATION SPEED COMPARISON

\begin{tabular}{r|ll}
\hline Frequency $f_{u}^{*}[1]$ & $\begin{array}{l}\text { Computation time [s] } \\
\text { in original representation }\end{array}$ & $\begin{array}{l}\text { Computation time }[\mathrm{s}] \\
\text { in proposed representation }\end{array}$ \\
\hline 0.001 & 6942 & 3131 \\
0.002 & 9028 & 4777 \\
0.003 & 8242 & 3909 \\
0.02 & 3295 & 1722 \\
0.1 & 1675 & 841 \\
0.3 & 1205 & 572 \\
0.5 & 695 & 486 \\
10 & 374 & 272 \\
\hline
\end{tabular}

proposed nondimensional representation has $O\left(n^{3}\right)+O\left(n^{5}\right)$ time complexity at maximum that is still better than $O\left(n^{8}\right)$ time complexity even at $n=2$. The parameter space reduction was also evaluated.

Table I evaluates the nonlinear dynamics computation time, which is the sum of all computation times within $D^{*} A^{*} P^{*}$ space needed to make Fig. 3. At every frequency $f_{u}^{*}$, as expected, the computation time for $x(t)=X_{s} \phi\left[\theta_{\text {special }}^{*}(\theta), x^{*}(0), u^{*}\left(t / T_{s}\right)\right]$ using the proposed nondimensional representation (3), (4) was better than that for $x(t)=\phi[\theta, x(0), u(t)]$ using the original representation (1), (2). In total, the computation time of the proposed nondimensional representation was reduced to $15710 \mathrm{~s}(4.2 \mathrm{~h})$, which is around half of that of the original representation $31455 \mathrm{~s}(8.7 \mathrm{~h})$. This is because parameters $\left(M, A_{+}, L, b, C\right)=(1,1,1,1,1)$ reduce the number of multiplication and division operations preserving the parametric structure in the original representation (1), (2). The computation time can be improved more since all existing methods [8], [33] developed for the original representation (1), (2) can be applied. While the computations were made for only eight frequencies $f_{u}^{*}$ in Table I, the fast computation was well evaluated.

Finally, let us remark that Proposition 1 and Theorem 1 provide the links to the closed-loop discussion as well as the openloop discussion presented in this paper.

Remark 3 (Relating to design and control via scaling): Let us put a simple example of the links based on our experimental 1-DOF arm, and consider a scaling design and control problem of a hydraulic cylinder whose piston undershoot should be zero in the presence of force disturbance. Assume that only $\left(D, A_{+}, b\right)=\left(11000 \mathrm{Ns} / \mathrm{m}, 0.0021 \mathrm{~m}^{2}, 5.3 \times 10^{8} \mathrm{~Pa}\right)$ are given and the others, $\left(M, L, A_{-}, C, P\right) \in \mathbb{R}_{+}^{5}$ and a gain $F>0$ of the simple control $u(t)=-F s(t)$, are unknown under a certain working constraints $L / 2 \geq 2.5 \mathrm{~m}$ (with pipeline length effect), $A_{-} \leq 0.0016 \mathrm{~m}^{2}, P \leq 21 \times 10^{6} \mathrm{~Pa}$ in the large scale.

Step 1: In the large scale, we search the parameters $\left(M, L, A_{-}, C, P\right)$ by the advanced direct search approach. Since the transfer function in the following linearizationbased control (the classical control) does not treat any initial response $\bar{x}(t):=\phi[\theta, x(0), u(t) \equiv 0]$, the objective function is the norm overshoot $\max _{0 \leq t<\infty}\left|\bar{x}(t)^{\mathrm{T}} Q \bar{x}(t)\right|$ by the random initial state $x(0) \in \Omega_{L P}$. Here, we will suffer from $O\left(n^{5}\right)$ time complexity without Proposition 1 and Theorem 1, but now only $O\left(n^{3}\right)$ time complexity is needed. When $n=10$ and $Q=\operatorname{diag}(1,1,0.1,0.1)$, the searched
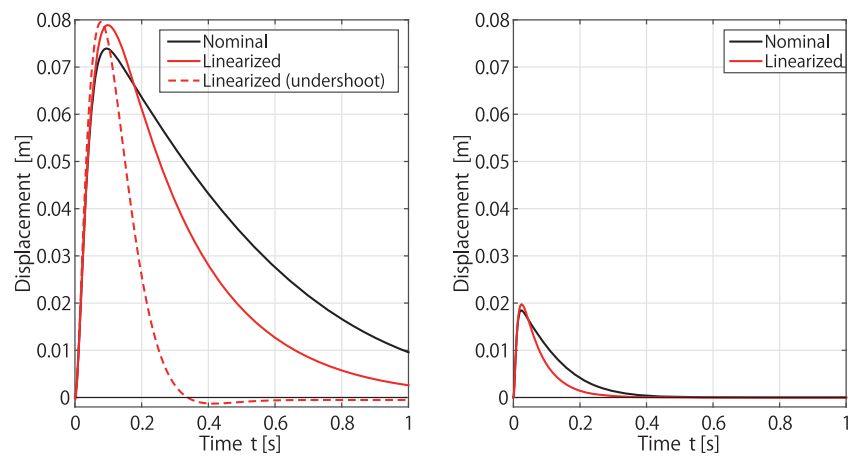

Fig. 6. Disturbance response (Left: large scale, Right: small scale).

parameters are $\left(D^{*}, A^{*}, P^{*}\right)=(4.1,0.75,0.028)$, which imply $\left(M, L, A_{-}, C, P\right)=\left(M, 0.144 M, 0.0016 \mathrm{~m}^{2}, C, 14 \times\right.$ $10^{6} \mathrm{~Pa}$ ) with free parameters $M$ and $C$. Under these constraints, our choice is $M=100 \mathrm{~kg}$ and $C=1.8 \times 10^{-4} \sqrt{\mathrm{m}^{5} / \mathrm{kg}}$. These physical units are unique and dropped in the following.

Step 2: In the large scale, we prepare the linearization-based control $u(t)=-F s(t)$ whose nondimensional version is $u^{*}\left(t^{*}\right)=-\left(1 / U_{s}\right) F\left(L s^{*}\left(t^{*}\right)\right)=: F^{*} s^{*}\left(t^{*}\right)$. By the standard linear analysis, if $F^{*}<\left\{-\left(2\left(D^{*}-6\left(1+A^{*}\right)\right)^{3 / 2}\right) / 27+\right.$ $\left.2\left(D^{*}\right)^{3} / 27-2 D^{*}\left(1+A^{*}\right) / 3\right\} / \sqrt{8 P^{*}} \approx 1.82$, the zeroundershoot is guaranteed for the linearized model (12), but not guaranteed for the nominal model due to the nonlinearity. Fig. 6 shows the disturbance responses (the solid black and red curves) with $F=0.0128\left(F^{*}=0.908<1.82\right)$ of the nominal model and that of the linearized model (12) against the step disturbance $20000 \mathrm{~N}$. Fortunately, without adjusting $F$, the zero-undershoot for the nominal model is confirmed numerically (not analytically), whereas the response (the dashed red curve) with $F=0.0345\left(F^{*}=2.45>1.82\right)$ has the nonzero (dangerous) undershoot for the linearized model (12). The response difference between the models with the same gain corresponds to the nonlinearity (the FIT ratio $\approx 0$ in the band $\left.f_{u}^{*}<0.02\right)$ in Fig. 3, which justifies to use the nominal model instead of the linearized model (12).

Step 3: In a certain small scale, we design and control the small scaled hydraulic cylinder since the similarity [34] is sometimes required to reduce the experimental cost in the large scale. Here, by replacing $M=100 \rightarrow 25$ and keeping $\left(D^{*}, A^{*}, P^{*}\right)$ and $F^{*}$, we have $\left(M, L, A_{-}, C, P\right)=(25,3.6,0.0016,1.8 \times$ $\left.10^{-4}, 14 \times 10^{6}\right)$ and $F=0.0512$. Against the nonlinearity, based on Proposition 1 and Theorem 1, the zero-undershoot is guaranteed even for the nominal model in the small scale. Indeed, Fig. 6 shows no undershoots in the disturbance responses with $F=0.0512$ of both models in the small scale. Now, we can start to construct the small-scaled hydraulic cylinder for the experimental validation.

\section{CONCLUSION}

This paper reveals that a nominal model of hydraulic cylinders has a new simplicity on the parametric structure that is more accurate or complex, and merely simple existing models do not have. Without loss of generality, only by changing the 
damping constant $D^{*}$, the rod area $A^{*}$, and the source pressure $P^{*}$ and assuming that all the other physical parameters are unity, any index, such as the numerical existence and nonlinearity, is visualized efficiently. Three parameters $D^{*}, A^{*}$, and $P^{*}$ correspond to the damping parameter $d^{*}$ of the mass-damper-spring, or to the Froude number Fr and the Reynolds number Re of the fluid system in Section I. This is an inevitable, unexpected, and economical result. Roughly speaking, Fig. 4 is the best possible result corresponding to the analytical (analytic) solution. In this sense, the comprehensive relations between the nonlinear dynamics and many physical parameters are clarified. Besides small and large scale experiments, one of the key future work is to improve the accuracy of the nominal model keeping the simplicity for control.

\section{REFERENCES}

[1] J. Mattila, J. Koivumaki, D. G. Caldwell, and C. Semini, "A survey on control of hydraulic robotic manipulators with projection to future trends," IEEE/ASME Trans. Mechatronics, vol. 22, no. 2, pp. 669-680, Aug. 2017.

[2] S. Chen et al., "Adaptive robust cascade force control of 1-DOF hydraulic exoskeleton for human performance augmentation," IEEE/ASME Trans. Mechatronics, vol. 22, no. 2, pp. 589-600, Apr. 2017.

[3] J. Yao, Z. Jiao, and D. Ma, "A practical nonlinear adaptive control of hydraulic servomechanisms with periodic-like disturbances," IEEE/ASME Trans. Mechatronics, vol. 20, no. 6, pp. 2752-2760, Dec. 2015.

[4] T. Boaventura et al., "On the role of load motion compensation in highperformance force control," in Proc. IEEE/RSJ Int. Conf. Intell. Robots Syst., 2012, pp. 4066-4071.

[5] M. Jelali and A. Kroll, Hydraulic Servo Systems. New York, NY, USA Springer, 2002.

[6] H. Merritt, Hydraulic Control Systems. Hoboken, NJ, USA: Wiley, 1967.

[7] S. Sakai, K. Osuka, T. Maekawa, and M. Umeda, "Robust control systems of a heavy material handling agricultural robot: A case study for initial cost problem," IEEE Trans. Control Syst. Technol., vol. 15, no. 6, pp. 10381048, Nov. 2007.

[8] S. Sakai, "Casimir based fast computation for hydraulic robot optimizations," in Proc. IEEE/RSJ Int. Conf. Intell. Robots Syst., 2013, pp. 2874-2881.

[9] I. Nezu and H. Nakagawa, Turbulence in Open Channel Flows. Boca Raton, FL, USA: CRC Press, 1993

[10] J. Nurmi and J. Mattila, "Detection and isolation of leakage and valve faults in hydraulic systems in varying loading condition, part 1: Global sensitivity analysis," Int. J. Fluid Power, vol. 12, pp. 41-51, 2011.

[11] M. Vilenius, "Application of sensitivity analysis to electrohydraulic position servos," ASME J. Dyn. Syst. Meas. Control, vol. 106, pp. 77-82, 1984.

[12] G. Grabmair and K. Schlacher, "Energy-based nonlinear control of hydraulically actuated mechanical systems," in Proc. 44th IEEE Conf. Decis. Control, 2005, pp. 7520-7525.

[13] A. Kugi and W. Kemmetmuller, "New energy-based nonlinear controller for hydraulic piston actuators," Eur. J. Control, vol. 10, pp. 163-173, 2004.

[14] P. Y. Li and M. R. Wang, "Natural storage function for passivity-based trajectory control of hydraulic actuators," IEEE/ASME Trans. Mechatronics, vol. 19, no. 3, pp. 1057-1068, Jun. 2014.

[15] S. Sakai and Y. Maeshima, "A new method for parameter identification for $N$-dof hydraulic robots," in Proc. IEEE Int. Conf. Robot. Autom., 2014 pp. 5983-5989.

[16] K. Zhou, J. C. Doyle, and K. Glover, Robust and Optimal Control. Upper Saddle River, NJ, USA: Prentice-Hall, 1996.

[17] A. J. van der Schaft, L2-Gain and Passivity Techniques in Nonlinear Control. London, U.K.: Springer-Verlag, 2000.

[18] E. Buckingham, "On physically similar systems," Phys. Rev., vol. 4, pp. 354-376, 1931.

[19] W. D. Curtis, J. David Logan, and W. A. Parker, "Dimensional analysis and the pi theorem," Linear Algebra Appl., vol. 47, pp. 117-126, 1982.

[20] K. Fujimoto and T. Sugie, "Canonical transformation and stabilization of generalized Hamiltonian systems," Syst. Cont. Lett., vol. 42, no. 3, pp. 217-227, 2001.
[21] J. Watton, Fluid Power Systems. Englewood Cliffs, NJ, USA: PrenticeHall, 1989.

[22] B. M. Maschke, A. J. van der Schaft, and P. C. Breedveld, "An intrinsic Hamiltonian formulation of network dynamics: Non-standard Poisson structures and gyrators," J. Franklin Inst., vol. 329, pp. 923-966, 1992.

[23] A. J. van der Schaft and B. M. J. Maschke, "The Hamiltonian formulation of energy conservation physical systems and external ports," Archivfur Electronic Ubertrangungstechnik, vol. 49, pp. 362-371, 1995.

[24] V. Duindam, A. Macchelli, S. Stramigioli, and H. Bruyninckx, Modeling and Control of Complex Physical Systems: The Port-Hamiltonian Approach. New York, NY, USA: Springer, 2009.

[25] S. Stramigioli, C. Secchi, A. van. der. schaft Schaft, and C. Fantuzzi, "Sampled data systems passivity and discrete port-Hamiltonian systems," IEEE Trans. Robot., vol. 21, no. 4, pp. 574-587, Aug. 2005.

[26] K. Fujimoto and T. Sugie, "Iterative learning control of Hamiltonian systems: I/O based optimal control approach," IEEE Trans. Autom. Control, vol. 48, no. 10, pp. 1756-1761, Oct. 2003.

[27] M. R. Sirouspour and S. E. Salcudean, "Nonlinear control of hydraulic robots," IEEE Trans. Robot. Autom., vol. 17, no. 2, pp. 173-182, Apr. 2001.

[28] S. Sakai, "A special structure preserving nondimensionalization of hydraulic rotational joints," in Proc. IEEE Int. Conf. Robot. Autom., 2017, pp. 26-32.

[29] T. Yoshikawa, Foundation of Robotics. Cambridge, MA, USA: MIT Press, 1990

[30] H. K. Khalil, Nonlinear Systems. Englewood Cliffs, NJ, USA: PrenticeHall, 1996.

[31] B. Heinrichs, N. Sepehri, and A. B. Thornton-Trump, "Position-based impedance control of an industrial hydraulic manipulator," IEEE Control Syst., vol. 17, no. 1, pp. 46-52, Feb. 1997.

[32] L. Ljung, System Identification: Theory for the User. Englewood Cliffs, NJ, USA: Prentice-Hall, 1999

[33] S. Sakai and S. Stramigioli, "Passivity based control of hydraulic robot arms using natural Casimir functions," in Proc. IEEE/RSJ Int. Conf. Intell. Robot. Syst., 2008, pp. 538-544.

[34] V. I. Arnold, Mathematical Methods of Classical Mechanics. New York, NY, USA: Springer-Verlag, 1989.

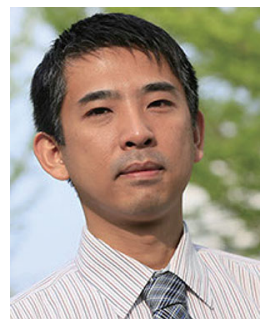

Satoru Sakai (M'17) received the B.E. degree in engineering and the M.E. and Ph.D. degrees in agricultural engineering from Kyoto University, Kyoto, Japan, in 1998, 2000, and 2003, respectively.

In 2005, he joined Chiba University, Chiba, Japan. In 2010, he joined Shinshu University, Matsumoto, Japan, where he is currently an Associate Professor with the Department of Mechanical Engineering. From 2003 to 2005, he was a JSPS Postdoctoral Research Fellow with the Department of Informatics, Kyoto University, and from 2004 to 2005 , he was a Visiting Researcher with the Faculty of Electrical Engineering, University of Twente, Enschede, The Netherlands. His research interests include robotics and systems and control.

Dr. Sakai was a recipient of the Young Author Prize at the International Federation of Automatic Control 2005.

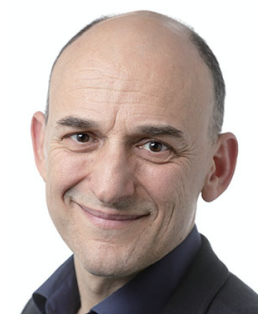

Stefano Stramigioli (F'15) received the M.Sc. (Hons.) degree (cum laude) from the University of Bologna, Bologna, Italy, in 1992, and the Ph.D. (Hons.) degree (cum laude) from the Delft University of Technology, Delft, The Netherlands, in 1998

$\mathrm{He}$ is currently a Full Professor in advanced robotics and the Chair Holder of the Robotics and Mechatronics Group, University of Twente, Enschede, The Netherlands. He has more than 200 publications including four books. He is currently the Vice-President for Research of euRobotics, the private part of the PPP with the EU known as SPARC.

Dr. Stramigioli has been an Officer and AdCom member for the IEEE/Robotics and Automation Society. 